\title{
Large odd-even radius staggering in the very light platinum isotopes from laser spectroscopy
}

\author{
F. Le Blanc, D. Lunney, ${ }^{*}$ J. Obert, J. Oms, J. C. Putaux, B. Roussière, J. Sauvage, and S. Zemlyanoi ${ }^{\dagger}$ \\ Institut de Physique Nucléaire, IN2P3-CNRS, F-91406 Orsay Cedex, France \\ J. Pinard, L. Cabaret, and H. T. Duong \\ Laboratoire Aimé Cotton, F-91405 Orsay Cedex, France \\ G. Huber, M. Krieg, and V. Sebastian \\ Institut für Physik der Universität Mainz, D-55099 Mainz, Germany \\ J. E. Crawford and J. K. P. Lee \\ Physics Department, McGill University, Montréal, Canada H3A 2T8 \\ M. Girod and S. Péru \\ Commissariat à l'Énergie Atomique, Service de Physique Nucléaire, BP 12, F-91680 Bruyères-le-Châtel, France \\ J. Genevey \\ Institut des Sciences Nucléaires, IN2P3-CNRS/UJF, F-38026 Grenoble Cedex, France
}

J. Lettry and ISOLDE Collaboration

CERN, CH-1211 Genève 23, Switzerland

(Received 28 April 1998; published 1 October 1999)

\begin{abstract}
Laser spectroscopy measurements have been carried out on very-neutron-deficient platinum isotopes with the COMPLIS experimental setup. Using the $5 d^{9} 6 s^{3} D_{3} \rightarrow 5 d^{9} 6 p^{3} P_{2}$ optical transition, hyperfine spectra of ${ }_{182,181,180,179,178} \mathrm{Pt}$ and ${ }^{183} \mathrm{Pt}^{m}$ were recorded for the first time. The variation of the mean square charge radius between these nuclei, the magnetic moments of the odd isotopes, and the quadrupole moment of ${ }^{183} \mathrm{Pt}^{m}$ were thus measured. A large deformation change between ${ }^{183} \mathrm{Pt}^{g}$ and ${ }^{183} \mathrm{Pt}^{m}$, an odd-even staggering of the charge radius, and a deformation drop from $A=179$ are clearly observed. Comparisons are made with microscopic theoretical predictions, in particular Hartree-Fock-Bogoliubov calculations using the Gogny force.

[S0556-2813(99)04810-4]
\end{abstract}

PACS number(s): 21.10.Ky, 21.10.Ft, 27.70.+q, 42.62.Fi

\section{INTRODUCTION}

The very neutron-deficient platinum isotopes belong to a mass region rich in shape instabilities. Along an isotopic chain, the crossing of the neutron midshell $N=104$ is accompanied by a number of fluctuations in nuclear shape. This is especially the case in the very-neutron-deficient mercury nuclei where a huge odd-even staggering of the nuclear charge radius between $N=106$ and $N=101$ was observed from laser spectroscopy $[1,2]$. This phenomenon has been interpreted as alternating oblate to prolate shape transitions between the even and the odd isotopes. Shape coexistence was first observed in ${ }^{186} \mathrm{Hg}[3,4]$ and further evidence of this phenomenon has been found in ${ }^{185} \mathrm{Hg}(N=105)$ where the ground state is prolate and the isomeric state oblate, thus explaining the very large difference measured in the charge radius [5].

\footnotetext{
*Permanent address: Centre de Spectrométrie Nucléaire et de Spectrométrie de Masse, IN2P3-CNRS, Université Paris-Sud, F-91405 Orsay Cedex, France.

${ }^{\dagger}$ Permanent address: Flerov Laboratory of Nuclear Reaction, JINR, Dubna RU-141980, Moscow Region, Russia.
}

This is in marked contrast to the behavior of the light platinum isotopes extensively studied by nuclear spectroscopy. In particular, all the low-lying energy levels in ${ }^{183} \mathrm{Pt},{ }^{181} \mathrm{Pt}$, and ${ }^{179} \mathrm{Pt}$ have been associated with a prolate shape [6-10] while shape coexistence is suggested for the lighter even ${ }^{172,174,176,178} \mathrm{Pt}$ isotopes [11-13]. However, laser spectroscopy studies previously performed on platinum isotopes from $A=193$ to $A=183$ [14-16], have shown a similar deformation of ${ }^{186} \mathrm{Pt}$ and ${ }^{185} \mathrm{Pt}^{m}[16,17]$ and a large radius change between ${ }^{186} \mathrm{Pt}$ and ${ }^{185} \mathrm{Pt}^{g}[15,17]$, suggesting shape coexistence in ${ }^{185} \mathrm{Pt}$. In addition, several studies of shape coexistence in the light platinum nuclei have shown the important impact of the presence of intruder states on the deformation of the ground and excited states [18-20]. The softness of $\mathrm{Pt}$ nuclei has also been extensively discussed $[15,16,21,22]$. It is therefore of main interest to study the behavior of the platinum far from stability by measuring (i) the change in the mean square charge radius $\left(\delta\left\langle r_{c}^{2}\right\rangle\right)$ in order to determine the deformation parameter of these exotic nuclei and (ii) the magnetic moment $\mu_{I}$ in order to confirm the neutron configuration of the odd isotopes. Moreover, the measurement of the quadrupole moment is crucial in order to determine the sign of the $\beta$ deformation for axially symmetric nuclei. 
We present high-precision optical spectroscopy measurements on very-neutron-deficient platinum isotopes. We recorded for the first time the hyperfine structure (HFS) of ${ }^{183 m}, 182-178 \mathrm{Pt}$. The HFSs of ${ }^{183} \mathrm{Pt}^{g}$ and ${ }^{185} \mathrm{Pt}^{g, m}$ were also more precisely measured. We thus extracted the isotope and isomeric shifts from which we determined the $\delta\left\langle r_{c}^{2}\right\rangle$ along this isotopic chain. In the odd isotopes we also extracted the magnetic $A_{i}$ hyperfine constants from which the $\mu_{I}$ values were deduced. In ${ }^{183} \mathrm{Pt}^{m}$ and ${ }^{185} \mathrm{Pt}^{g}$, we measured the electrostatic $B_{i}$ hyperfine constants to determine the spectroscopic quadrupole moments $Q_{s}$. Since platinum is refractory, such measurements are impossible at a standard ISOLbased facility without a secondary beam obtained from radioactive mercury decay. We used the COMPLIS experimental setup [23] which is especially designed for the study of such descendant elements.

In Sec. II, we present the experimental conditions used for the study of the platinum nuclei and the results we obtained for the very-neutron-deficient isotopes (from ${ }^{185} \mathrm{Pt}$ to ${ }^{178} \mathrm{Pt}$ ).

Section III is dedicated to a discussion of the nuclear moments and the mean square charge radius change in the light isotopes only, since the heavier ones have already been extensively discussed in Refs. [15] and [16]. Microscopic calculations are used to try to interpret the large odd-even staggering occurring between $A=185$ and $A=178$.

\section{EXPERIMENTAL METHOD AND RESULTS}

The experiment was performed on line with the PSBooster ISOLDE mass separator at CERN. The experimental procedure is the same as the one described in Ref. [24]: the mercury ions are slowed from $60 \mathrm{kV}$ to $1 \mathrm{kV}$, deposited on a graphite substrate, and desorbed as atoms after accumulation as granddaughter isotopes. They are then ionized in three atomic steps by a set of three pulsed tunable dye laser beams where the first laser excitation step at $306.5 \mathrm{~nm}\left(5 d^{9} 6 s^{3} D_{3}\right.$ $\left.\rightarrow 5 d^{9} 6 p^{3} P_{2}\right)$ is obtained from frequency doubling. The ions are finally detected with time-of-flight mass identification using a microchannel plate detector. ${ }^{185} \mathrm{Pt}^{g, m},{ }^{183} \mathrm{Pt}^{g, m}$, and ${ }^{182} \mathrm{Pt}$ were obtained via $\beta^{+} /[$electron capture (EC) $]$decay of $\mathrm{Hg}$. For the lighter isotopes, we are able to use the $\alpha$ decay mode to perform our measurements. So far from stability, the ISOLDE production yield is too low to produce them in sufficient quantities via successive $\beta$ decays. For example, the yield drops to 38 atoms/s for ${ }^{178} \mathrm{Hg}$ compared to 4 $\times 10^{6}$ atoms/s for ${ }^{182} \mathrm{Hg}$. With the $\alpha$ branching ratio of ${ }^{182} \mathrm{Hg}(15.2 \%)$ we obtain $8 \times 10^{6}{ }^{178} \mathrm{Pt}$ nuclei from $\alpha$ decay after $40 \mathrm{~s}$ collection. With the $\beta^{+} / \mathrm{EC}$ branching ratios of ${ }^{178} \mathrm{Hg}$ and ${ }^{178} \mathrm{Au}\left(30 \%\right.$ and $60 \%$, respectively), only $75{ }^{178} \mathrm{Pt}$ nuclei from $\beta^{+} / \mathrm{EC}$ decay would have been obtained during the same collection time, which is 100000 times less than from $\alpha$ decay. However, the desorption efficiency is slightly lower since after an $\alpha$ decay, a nucleus of a mass 180 recoils isotropically at about $120 \mathrm{keV}$ and can move out of the desorption area. To compare the efficiency, we recorded the hyperfine spectrum of ${ }^{180} \mathrm{Pt}$ obtained from the two types of radioactive disintegration, one by collecting ${ }^{180} \mathrm{Hg}$ and the other by collecting ${ }^{184} \mathrm{Hg}$. Counting the number of ${ }^{180} \mathrm{Pt}$ isotopes in resonance for each case, the ratio (number of de-

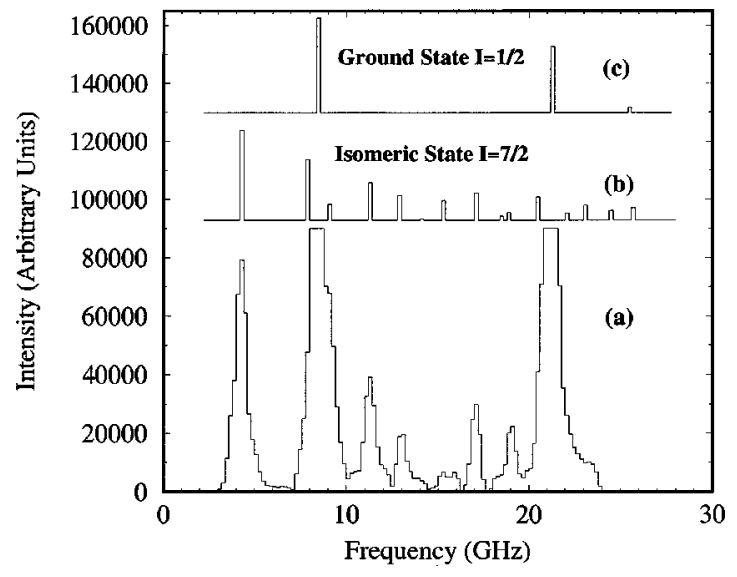

FIG. 1. (a) Experimental hyperfine spectrum for the $5 d^{9} 6 s^{3} D_{3}$ $\rightarrow 5 d^{9} 6 p^{3} P_{2}$ transition of ${ }^{183} \mathrm{Pt}^{g, m}$. The vertical scale is saturated for the two strongest peaks (corresponding to the ground state) in order to distinguish the weaker lines corresponding to the isomeric state. The two theoretical spectra above the experimental one have been reconstituted from the extracted hyperfine constants and the isomeric shift for the (b) isomeric and (c) ground states. The laser scan range is $24 \mathrm{GHz}$.

tected ions)/(number of collected ions) was smaller by a factor of 5 for the $\alpha$ decay. Even though the efficiency is lowered with $\alpha$ decay, this constitutes a new application of our experimental technique that allows access to nuclei even further from stability.

The HFS and isomeric shift of ${ }^{183} \mathrm{Pt}^{g, m}$ obtained from the $306.5 \mathrm{~nm}$ resonant transition are shown as an example in Fig. 1. The two intense lines can be easily identified as those of the ground state since its spin is $1 / 2$. With its $7 / 2$ spin value, ${ }^{183} \mathrm{Pt}^{m}$ distributes its intensity over some ten main lines. Moreover, since the half-lives of the two states are very different $\left[T_{1 / 2}^{m}=43(5) \mathrm{s}\right.$ and $\left.T_{1 / 2}^{g}=6.5(10) \mathrm{m}\right]$, the ratio of ground state to isomer nuclei varies from 2.5 to 7 depending on the measurement delay times (57 s and $172 \mathrm{~s}$, respectively).

The magnetic moments of the odd isotopes are obtained from $A_{i}$ of the atomic ground state and the precisely known value of $\mu_{I}$ of ${ }^{195} \mathrm{Pt}[25,26]$

$$
\mu_{I}\left({ }^{x} \mathrm{Pt}\right)=0.214(2) A_{i}\left({ }^{x} \mathrm{Pt}\right) I\left({ }^{x} \mathrm{Pt}\right)
$$

the error being due to $1 \%$ hyperfine anomaly uncertainty. The $\mu_{I}$ values are presented in Table I. The two values measured by Hilberath et al. [16] (for ${ }^{185} \mathrm{Pt}^{m}$ and ${ }^{183} \mathrm{Pt}^{g}$ ) are consistent with our measurements.

For ${ }^{183} \mathrm{Pt}^{m}$ and ${ }^{185} \mathrm{Pt}^{g}$ the $B_{i}$ factors of the ${ }^{3} D_{3}$ atomic ground state have been extracted from the hyperfine spectra. $Q_{s}$ is related to $B_{i}$ via

$$
Q_{s}\left({ }^{x} P t\right)=-0.685 B_{i}\left({ }^{x} \mathrm{Pt}\right)
$$

which is obtained from the calculated electric field gradient as described in Ref. [16]. The $Q_{s}$ values have then to be corrected for the Sternheimer shielding factor $R_{5 d}$. This factor, obtained for platinum from systematics on the $5 d$ shell, 
TABLE I. Nuclear moments of the odd Pt isotopes.

\begin{tabular}{ccccc}
\hline \hline$A$ & $I^{\pi}$ & $\mu_{I}\left[\mu_{N}\right]$ & $Q_{s}[\mathrm{~b}]$ & $Q_{s, \text { corr }}[\mathrm{b}]$ \\
\hline $185^{g}$ & $9 / 2^{+}$ & $-0.723(11)$ & $+4.10(19)$ & $+3.73(17)$ \\
$185^{m}$ & $1 / 2^{-}$ & $+0.503(5)$ & & \\
$183^{g}$ & $1 / 2^{-}$ & $+0.502(5)$ & & \\
$183^{m}$ & $7 / 2^{-}$ & $+0.782(14)$ & $+3.71(30)$ & $+3.37(27)$ \\
181 & $1 / 2^{-}$ & $+0.484(21)$ & & \\
179 & $1 / 2^{-}$ & $+0.431(32)$ & & \\
\hline \hline
\end{tabular}

lowers the measured $Q_{s}$ by $9.1 \%$ [27]. The corrected and measured $Q_{s}$ values are presented in Table I.

The experimental isotope shift (IS) values relative to ${ }^{194} \mathrm{Pt}$ are given in Table II. The IS consists of a mass shift $\delta \nu_{\text {MS }}^{A, A^{\prime}}$ and a field shift $\delta \nu_{\mathrm{FS}}^{A, A^{\prime}}$ [29]. For the heavy elements, the mass shift contribution is small. For an $n s \rightarrow n p$ transition, it has been estimated to be $(1.3 \pm 0.9) \Delta \nu_{\mathrm{NMS}}$ where $\Delta \nu_{\mathrm{NMS}}$ is the easily calculable normal mass shift [29]. The nuclear parameter $\lambda^{A, A^{\prime}}$ related to the change in the nuclear charge radius is extracted from the remaining $\delta \nu_{\mathrm{FS}}^{A, A^{\prime}}$ using $\delta \nu_{\mathrm{FS}}^{A, A^{\prime}}$ $=F_{306} \lambda^{A, A^{\prime}}$, where $F_{306}$ is the electronic factor of the atomic transition. Since no relativistic multiconfiguration DiracFock (MCDF) calculations have been performed for this wavelength, we derived this factor from a King plot made on the stable isotopes as described in Ref. [30], using the calculated $F_{266}$ value of the $266 \mathrm{~nm}$ transition [16] and the experimental IS data of both transitions. This leads to $F_{306}$ $=-18.5(10) \mathrm{GHz} / \mathrm{fm}^{2}$. It is thus possible to extract $\delta\left\langle r_{c}^{2}\right\rangle^{194, A}$ following the method described in Refs. [31,32] and the mean square deformation parameter variation $\delta\left\langle\beta^{2}\right\rangle^{194, A}$ can be deduced. All those values are given in Table II.

Plotted in Fig. 2 are the measured $\delta\left\langle r_{c}^{2}\right\rangle$ for the platinum isotopes. The results obtained are in agreement with those of Refs. $[15,16]$ and the error bars have been considerably reduced especially for ${ }^{183} \mathrm{Pt}$. One can make the following re-

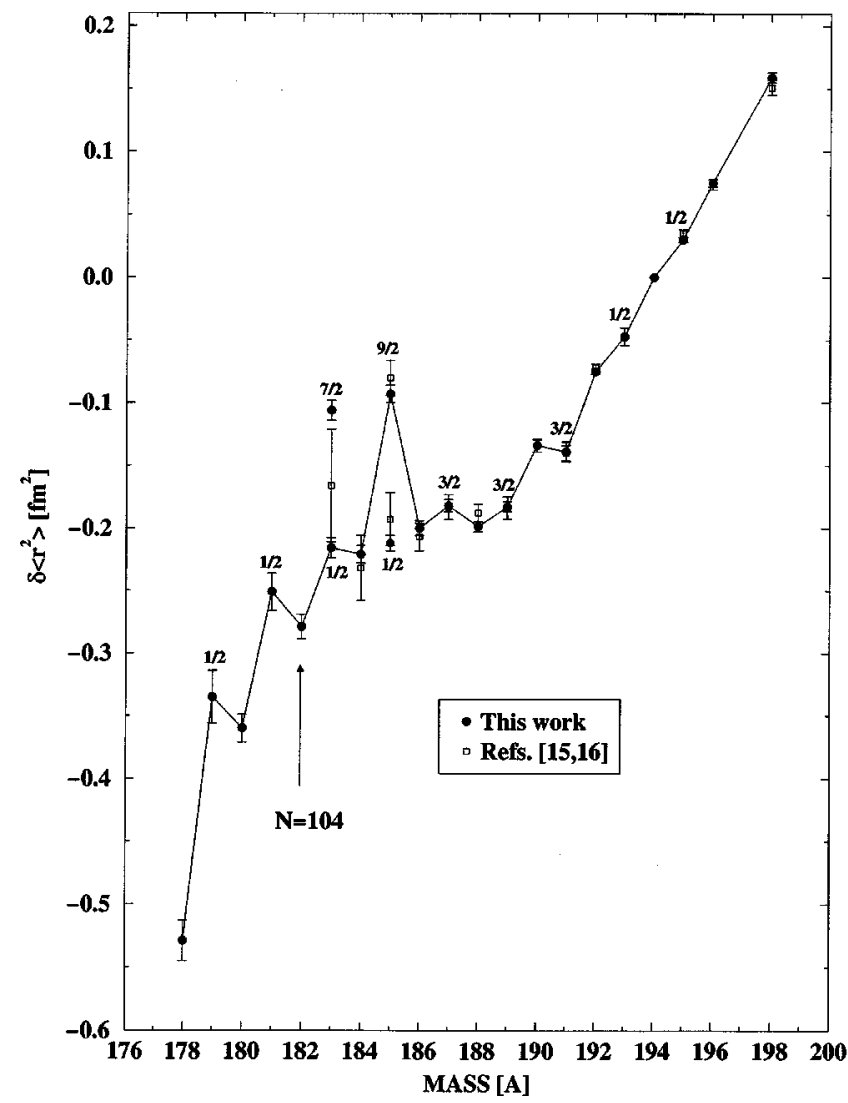

FIG. 2. Mean square charge radius variation of the platinum isotopes (this work and Refs. $[15,16])$. The number above each odd mass represents the spin of the state. The ground states of the nuclei have been linked to emphasize the odd-even staggering.

marks: (i) there is a large difference in the radius of the ground and isomeric states for both ${ }^{185} \mathrm{Pt}$ and ${ }^{183} \mathrm{Pt}$, (ii) there is a marked decrease of the radius and thus of the deformation at mass 178, and (iii) there is quite a strong inverted odd-even staggering (OES) below mass 188 in contrast with the small OES observed above.

TABLE II. Mean square charge radius variation in light platinum isotopes. ${ }^{194} \mathrm{Pt}$ is taken as the reference isotope for $\delta\left\langle r_{c}^{2}\right\rangle$. The $B(E 2)$ value of ${ }^{194} \mathrm{Pt}$ [28] is used as a reference to extract the rms deformation parameters $\left\langle\beta^{2}\right\rangle^{1 / 2}$.

\begin{tabular}{cccccc}
\hline \hline$A$ & $I^{\pi}$ & $\delta \nu^{194, A}[\mathrm{GHz}]$ & $\delta\left\langle r_{c}^{2}\right\rangle^{194, A}\left[\mathrm{fm}^{2}\right]$ & $\delta\left\langle\beta^{2}\right\rangle^{194, A}$ & $\left\langle\beta^{2}\right\rangle^{1 / 2}$ \\
\hline $185^{g}$ & $9 / 2^{+}$ & $1.582(35)$ & $-0.093(7)$ & $0.0330(6)$ & $0.231(3)$ \\
$185^{m}$ & $1 / 2^{-}$ & $3.611(7)$ & $-0.212(6)$ & $0.0220(5)$ & $0.207(3)$ \\
184 & 0 & $3.76(6)$ & $-0.221(7)$ & $0.0259(6)$ & $0.216(3)$ \\
$183^{g}$ & $1 / 2^{-}$ & $3.67(5)$ & $-0.216(8)$ & $0.0308(7)$ & $0.227(3)$ \\
$183^{m}$ & $7 / 2^{-}$ & $1.81(4)$ & $-0.106(8)$ & $0.0403(7)$ & $0.246(3)$ \\
182 & 0 & $4.75(8)$ & $-0.279(10)$ & $0.0298(9)$ & $0.225(4)$ \\
181 & $1 / 2^{-}$ & $4.27(20)$ & $-0.251(15)$ & $0.0367(13)$ & $0.239(4)$ \\
180 & 0 & $6.13(5)$ & $-0.360(11)$ & $0.0318(9)$ & $0.229(4)$ \\
179 & $1 / 2^{-}$ & $5.70(30)$ & $-0.335(21)$ & $0.0384(18)$ & $0.243(5)$ \\
178 & 0 & $8.99(20)$ & $-0.529(16)$ & $0.0261(14)$ & $0.216(5)$ \\
\hline \hline
\end{tabular}


TABLE III. $\beta$ parameters extracted from the hyperfine structure and from the isotope shift.

\begin{tabular}{cccccc}
\hline \hline$A$ & $I^{\pi}$ & $Q_{s, \text { corr }}[\mathrm{b}]$ & $Q_{0}[\mathrm{~b}]$ & $\beta$ & $\left\langle\beta^{2}\right\rangle^{1 / 2}$ \\
\hline $185^{g}$ & $9 / 2^{+}$ & $+3.73(17)$ & $+6.84(31)$ & $+0.229(10)$ & $0.231(3)$ \\
$183^{m}$ & $7 / 2^{-}$ & $+3.37(27)$ & $+7.22(58)$ & $+0.242(18)$ & $0.246(3)$ \\
\hline \hline
\end{tabular}

\section{DISCUSSION}

\section{A. Nuclear moments}

To extract the intrinsic quadrupole moment $Q_{0}$ of ${ }^{185} \mathrm{Pt}^{g}$ and ${ }^{183} \mathrm{Pt}^{m}$, we have assumed axial symmetry and a strong coupling scheme using the expression

$$
Q_{0}=Q_{s}^{\text {corr }} \frac{(I+1)(2 I+3)}{3 K^{2}-I(I+1)},
$$

where $I$ is the nuclear spin and $K$ the projection of $I$ on the nuclear symmetry axis. The $\beta$ deformation parameter can thus be extracted using the expression

$$
Q_{0}=\frac{3}{\sqrt{5 \pi}} Z R_{0}^{2} \beta\left(1+\frac{2}{7} \sqrt{\frac{5}{\pi}} \beta\right) \text {. }
$$

Table III represents the deformation parameter of the two nuclei extracted by the two methods: one using $\delta\left\langle r_{c}^{2}\right\rangle$ obtained from the isotope shift and the other using $Q_{s}$ extracted from the hyperfine structure if we suppose axial symmetry. The two values of the deformation for each nucleus are very close which means that the axial symmetry assumed is justified. The presence of $\gamma$ asymmetry would involve a $K$ mixture in the wave function. Thus, Eq. (1) cannot be applied and consequently the $\beta$ value would be different from the $\left\langle\beta^{2}\right\rangle^{1 / 2}$ value extracted from the isotope shift. Therefore, it is clearly shown that these two nuclei are axially prolate shaped.

Moreover, the measured $Q_{s}$ of Table III partially answers the question addressed in Ref. [16] as to whether or not $\gamma$ asymmetry is present in the odd isotopes below $A=185$. Indeed, the total Routhian surface (TRS) calculations predicted well the axiality of these two nuclei whereas the partical triaxial rotor (PTR) ones predicted $\gamma=22^{\circ}$ for ${ }^{185} \mathrm{Pt}^{g}$ and $\gamma$ $=20^{\circ}$ for ${ }^{183} \mathrm{Pt}^{m}$.
The values of $\mu_{I}$ are presented and compared with theoretical predictions in Table IV. The magnetic moments have been calculated in the framework of the axial rotor +quasiparticle coupling model in which the quasiparticle wave functions are self-consistently obtained for a neighboring even core as described in Refs. [33,34]. In the present work, the core has been constrained to take the $\left\langle\beta^{2}\right\rangle^{1 / 2}$ deformation extracted from our $\delta\left\langle r_{c}^{2}\right\rangle$ measurements of the odd nuclei (see Table II). In Table IV, we have also included the $\mu_{I}$ values calculated by Hilberath et al. [16]. The two types of calculations are in good agreement and one observes almost no differences in the calculations of Ref. [16] whether $\gamma$ is equal to zero or not (see columns 5 and 6). This shows that the magnetic moment is not very sensitive to $\gamma$. Thus, a prolate shape for all the states is not in contradiction with the axial symmetry found for ${ }^{185} \mathrm{Pt}^{g}$ and ${ }^{183} \mathrm{Pt}^{m}$ from the $Q_{s}$ values.

\section{B. Mean square charge radius variation}

Figure 3(a) presents the systematics of the low-lying energy levels of the even-even isotopes. The state energies of the ground state band decrease down to $A=186$, are constant until $A=178$, and increase at lower $A$. In Fig. 3(b), the charge radius decreases regularly down to $A=188$ where it becomes rather smooth and then sharply decreases between $A=180$ and $A=178$. The variation of the moment of inertia of the ground state band (deduced from the energy levels) and the mean square charge radius variation both indicate a sharp deformation change between ${ }^{188} \mathrm{Pt}$ and ${ }^{186} \mathrm{Pt}$ and around ${ }^{178} \mathrm{Pt}$. Moreover, the presence of a second $2^{+}$state at low energy is a strong indication of triaxiality for all these even nuclei.

The issue is now to try to interpret the large odd-even staggering occurring below mass 186. This OES is found to be inverted which is not usually the case. Generally, the even nuclei are found to be more deformed than their odd neighbors and the odd-even staggering is called "normal." The normal OES is associated with pairing effects which increase $\left\langle r_{c}^{2}\right\rangle$ for the even-even nuclei [35].

We have carried out Hartree-Fock-Bogoliubov (HFB) calculations using the Gogny force [36,37] to compare the predictions of the model with the experimental $\delta\left\langle r_{c}^{2}\right\rangle$ values. These calculations are parameter free. This means that no adjustment can be made from one isotope to another to im-

TABLE IV. Experimental and theoretical magnetic moments of the odd Pt isotopes.

\begin{tabular}{cccccc}
\hline \hline$A$ & State & $\mu_{\exp }\left[\mu_{N}\right]$ & $\mu_{\text {cal }}{ }^{\mathrm{a}}\left[\mu_{N}\right]$ & $\mu_{\text {cal }}{ }^{\mathrm{b}}\left[\mu_{N}\right]$ & $\mu_{\text {cal }}{ }^{\mathrm{c}}\left[\mu_{N}\right]$ \\
\hline $185^{g}$ & $9 / 2^{+}[624]$ & $-0.723(11)$ & $-1.46(-0.86)$ & -0.90 & -0.93 \\
$185^{m}$ & $1 / 2^{-}[521]$ & $+0.503(5)$ & $+0.63(+0.37)$ & +0.47 & +0.46 \\
$183^{g}$ & $1 / 2^{-}[521]$ & $+0.502(5)$ & $+0.63(+0.37)$ & +0.47 & +0.45 \\
$183^{m}$ & $7 / 2^{-}[514]$ & $+0.782(14)$ & $+1.45(+0.98)$ & +0.91 & +0.86 \\
181 & $1 / 2^{-}[521]$ & $+0.484(21)$ & $+0.63(+0.38)$ & +0.47 & +0.45 \\
179 & $1 / 2^{-}[521]$ & $+0.431(32)$ & $+0.64(+0.38)$ & & \\
\hline \hline
\end{tabular}

${ }^{\mathrm{a}}$ Our axial rotor and quasiparticle calculations for $g_{s}=g_{s}$ free $\left(0.6 g_{s}\right.$ free $)$.

${ }^{\mathrm{b}}$ The particle triaxial rotor calculations from Ref. [16] for $g_{s}=0.2 g_{s}$ free and $\gamma \neq 0^{\circ}$.

${ }^{\mathrm{c}}$ The particle triaxial rotor calculation from Ref. [16] for $g_{s}=0.7 g_{s}$ free and $\gamma=0^{\circ}$. 

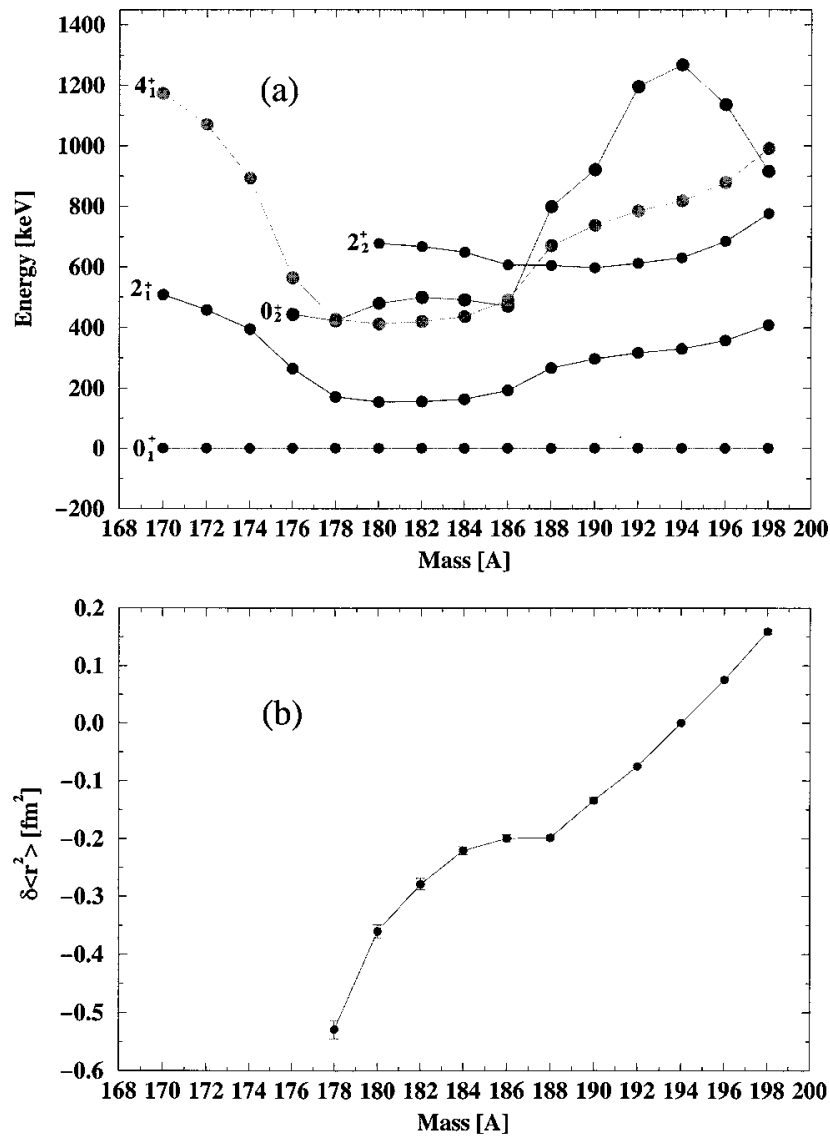

FIG. 3. Evolution of the low-lying $0_{1}^{+}, 2_{1}^{+}, 0_{2}^{+}, 4_{1}^{+}$, and $2_{2}^{+}$ energies (a) and mean square charge radius variation (b) through the range $A=170-198$ of the even isotopes.

prove the agreement between theory and experiment. All the multipole operator mean values $\left(\beta_{2}, \beta_{4}, \beta_{6}, \ldots\right)$ are selfconsistently obtained by a minimization process.

In a first step, we have performed constrained HFB calculations with respect to axial deformation $\beta$ to deduce the potential energy curves (PECs) $V(\beta)=\left\langle\Phi_{\beta}|\hat{H}| \Phi_{\beta}\right\rangle$, where $\hat{H}$ is the nuclear Hamiltonian and $\left|\Phi_{\beta}\right\rangle$ the quasiparticle (qp) vacuum.

For the even nuclei these PECs clearly show a shape transition from oblate shapes for the heavy isotopes to prolate shapes for the light ones. This feature indicates that the triaxial degree of freedom plays an important role, especially in the vicinity of the shape transition near $A=186$ where the oblate and prolate minima are almost degenerate as is shown in Fig. 4. For the odd nuclei, we have extended the HFB formalism using blocking techniques. In this context the ground state wave functions for even-odd nuclei are expressed as

$$
|\widetilde{\Phi}\rangle=\eta_{b}^{+}|\Phi\rangle,
$$

where $|\Phi\rangle$ is the HFB vacuum of the even-even core, $\eta^{+}$a $q p$ creation operator, and $b$ is the label for the quantum numbers of the blocked quasiparticles. Since axial symmetry is imposed, $b=(\Omega, \pi)$, where $\Omega$ is the projection of the angular momentum on the $z$ axis and $\pi$ the parity. Then by per-

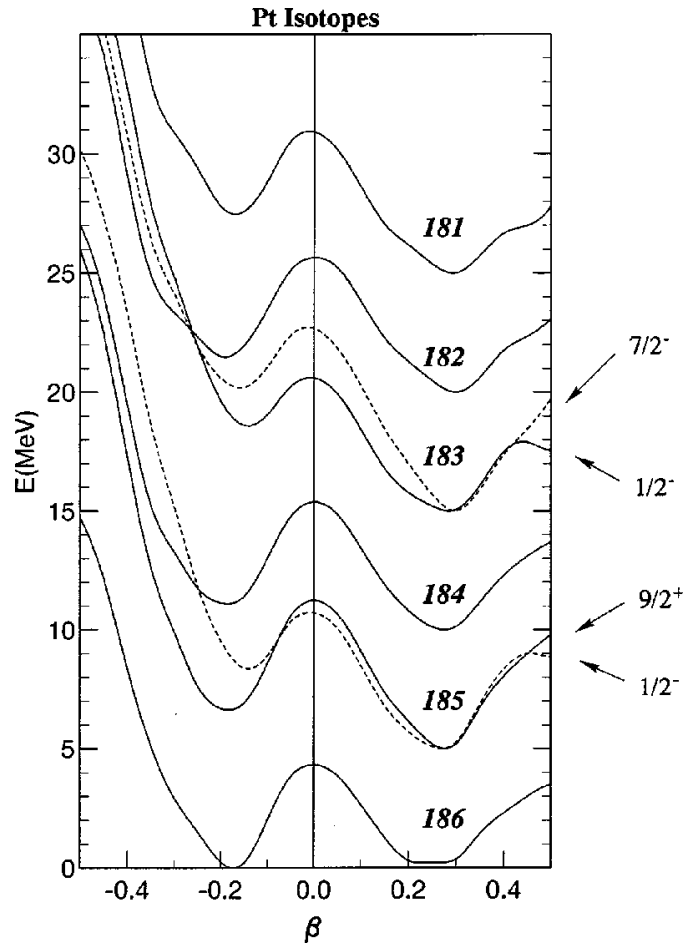

FIG. 4. Potential energy curves for ${ }^{181-186} \mathrm{Pt}$ obtained with axial Hartree-Fock-Bogoliubov calculations. To make the comparison between predictions easier, the PECs values are presented relative to their respective absolute minima. These minima are shifted by $+5 \mathrm{MeV}$ when moving from one isotope to the lower $A$.

forming self-consistent blocking HFB calculations with various quasiparticle states, we obtain energy spectra. The ground state of an odd nucleus is determined by finding the blocked state $b$ which minimizes the total energy. Constrained HFB calculations using this blocking procedure have been performed for the odd Pt isotopes. As shown in Fig. 4, the curve $V(\beta)=\left\langle\widetilde{\Phi}_{\beta}|\hat{H}| \widetilde{\Phi}_{\beta}\right\rangle$ shows larger oblateprolate energy differences for odd isotopes than for the even ones, especially in the vicinity of the shape transition $(A$ $\simeq 186$ ). This indicates that for these odd nuclei, the unpaired particle favors axial symmetry, either oblate or prolate, in such a way that the triaxial degree of freedom would play a minor role in the nuclear dynamics. Furthermore, two sets of PECs are shown for ${ }^{183} \mathrm{Pt}$ and ${ }^{185} \mathrm{Pt}$ : one for their respective $1 / 2^{-}$and $9 / 2^{+}$ground states (solid curves) and the other one for their respective $7 / 2^{-}$and $1 / 2^{-}$isomeric states (dashed curves). They show that a larger deformation is predicted for the $7 / 2^{-}$and $9 / 2^{+}$states than for the two $1 / 2^{-}$states.

In the second step, we have performed full triaxial HFB calculations [38] for the even platinum $(A=176-196)$ isotopes. Once the constrained HFB equations are solved, the potential energy surface (PES) is defined as

$$
V(q)=\left\langle\Phi_{q}|\hat{H}| \Phi_{q}\right\rangle,
$$

where the notation $q=\left(q_{20}, q_{22}\right)$ means that constraints are made on the quadrupole moment components. Since $q_{20}$ and $q_{22}$ are directly related to the Bohr coordinates $\beta$ and $\gamma$, the potential energy surface (2) may also be expressed as 
$V(\beta, \gamma)$. Among these surfaces, those with $A<188$ are soft with respect to $\gamma$ deformation. For ${ }^{188} \mathrm{Pt}$, the absolute PES minimum is triaxial.

Going a step beyond the mean field theory, we have performed configuration mixing calculations in the framework of the generator coordinate method (GCM) assuming the Gaussian overlap approximation (GOA) [39]. This approach takes into account the rotation-vibration coupling which is expected to be strong in soft nuclei. In this theory, the dynamical states (i.e., the ground state and excited levels) are sought as

$$
|\Psi\rangle=\int f(q)\left|\Phi_{q}\right\rangle d q,
$$

where the superposition amplitude $f(q)$ is solution of the Griffin-Hill-Wheeler equation [40]. The GOA is obtained by assuming that the overlap kernel $I\left(q, q^{\prime}\right)=\left\langle\Phi_{q} \mid \Phi_{q^{\prime}}\right\rangle$ entering the Hill-Wheeler equation has a Gaussian shape. Under this approximation, this equation can be transformed into a second-order differential equation and expressed in the laboratory system

$$
\hat{\mathcal{H}} g(q)=E g(q),
$$

where

$$
\hat{\mathcal{H}}=-\frac{\hbar^{2}}{2} \sum_{i, j} \frac{\partial}{\partial q_{i}}\left(M^{-1}(q)\right)_{i j} \frac{\partial}{\partial q_{j}}+\mathcal{V}(q)
$$

is the collective Hamiltonian, $g(q)$ the Gauss transform of $f(q)$, and $\mathcal{V}(q)$ the potential energy surface corrected for zero-point energy $\Delta V(q)$ [i.e., $\mathcal{V}(q)=V(q)-\Delta V(q)$ ]. This correction term $\Delta V(q)$ and the collective masses $B_{i j}(i, j$ $=0,2)$ and moments of inertia and $J_{i}(i=1,3)$ which completely define the tensor $M_{i j}$ are calculated in the cranking approximation [41].

Solving $\hat{\mathcal{H}}$ provides us the ground state collective wave functions. With these wave functions, we calculate root mean square beta and gamma deformations ( $\beta_{\mathrm{rms}}$ and $\gamma_{\mathrm{rms}}$, respectively) and mean square charge radii

$$
\left\langle r_{c}^{2}\right\rangle_{\mathrm{th}}=\left\langle\widetilde{\Psi}_{0}\left|\hat{r}^{2}\right| \Psi_{0}\right\rangle /\left\langle\widetilde{\Psi}_{0} \mid \widetilde{\Psi}_{0}\right\rangle,
$$

where $\left|\widetilde{\Psi}_{0}\right\rangle$ is the ground state collective wave function here defined as $\left|\widetilde{\Psi}_{0}\right\rangle=\int g(q)\left|\Phi_{q}\right\rangle d q$.

Table $\mathrm{V}$ includes $\beta_{\mathrm{rms}}$ and $\gamma_{\mathrm{rms}}$ values for the ground state of even-even isotopes together with the experimental and calculated $\delta\left\langle r_{c}^{2}\right\rangle^{194, A}$ values. Figure 5 displays a comparison between these experimental and calculated values. Recently Esser et al. [42] extracted the $\gamma$ and $\beta$ deformation parameters from $B(E 2)$ measurements and from $2_{1}^{+}$and $2_{2}^{+}$energies, for the even isotopes ${ }^{180-198} \mathrm{Pt}$. Even though the present rms $\gamma$ deformations are slightly lower and the rms $\beta$ deformations a bit larger than the ones obtained by Esser et al., the general trend of the $\delta\left\langle r_{c}^{2}\right\rangle$ measurement is reproduced, in particular the kink appearing at mass 188 . However, the difference between theory and experiment is larger for lighter isotopes. This is partly due to the arbitrary choice of ${ }^{194} \mathrm{Pt}$ as
TABLE V. Calculated $\beta_{\mathrm{rms}}$ and $\gamma_{\mathrm{rms}}$ deformation parameters of the even-even isotopes. Experimental and calculated $\delta\left\langle r_{c}^{2}\right\rangle^{194, A}$ values are also shown.

\begin{tabular}{ccccc}
\hline \hline$A$ & $\beta_{\mathrm{rms}}$ & $\gamma_{\mathrm{rms}}(\mathrm{deg})$ & $\delta\left\langle r_{c}^{2}\right\rangle_{\mathrm{th}}^{194, A}\left[\mathrm{fm}^{2}\right]$ & $\delta\left\langle r_{c}^{2}\right\rangle_{\mathrm{expt}}^{194, A}\left[\mathrm{fm}^{2}\right]$ \\
\hline 196 & 0.14 & 33 & 0.109 & $0.075(3)$ \\
194 & 0.15 & 31 & 0 & 0 \\
192 & 0.17 & 31 & -0.098 & $-0.075(2)$ \\
190 & 0.18 & 29 & -0.190 & $-0.134(5)$ \\
188 & 0.20 & 25 & -0.228 & $-0.199(3)$ \\
186 & 0.24 & 19 & -0.169 & $-0.200(6)$ \\
184 & 0.27 & 15 & -0.119 & $-0.221(7)$ \\
182 & 0.29 & 13 & -0.127 & $-0.279(10)$ \\
180 & 0.31 & 12 & -0.161 & $-0.360(11)$ \\
178 & 0.31 & 13 & -0.313 & $-0.529(16)$ \\
176 & 0.30 & 13 & -0.518 & \\
174 & 0.25 & 16 & -0.924 & \\
\hline \hline
\end{tabular}

the reference nucleus. Moreover, it can be observed that, in this mass region, $\beta$ deformations are overestimated as one goes further from stability. This can be due to the parameters of the force which are fitted to the properties of only a few spherical nuclei of the nuclide chart. This overestimation of $\beta$ can explain the discrepancy observed below $A=187$ in the $\delta\left\langle r_{c}^{2}\right\rangle$ values. Nevertheless, one must stress that the deviation between measured and calculated $\delta\left\langle r_{c}^{2}\right\rangle$ values never exceeds $0.25 \mathrm{fm}^{2}$, which is modest for microscopic calculations free of adjustable parameters.

Since $\Omega$ is no longer a good quantum number when axial symmetry is broken, we cannot perform triaxial HFB and GCM calculations for odd isotopes. From now on, we limit our description of even-odd Pt isotopes by assuming axial symmetry in the calculations. In fact, this limitation is generally not too drastic. Both experimental and theoretical arguments lead us to believe that this limitation is reasonable. The experimental argument has been given in Sec. III A: the quadrupole moment values measured for ${ }^{185} \mathrm{Pt}^{g}$ and ${ }^{183} \mathrm{Pt}^{m}$

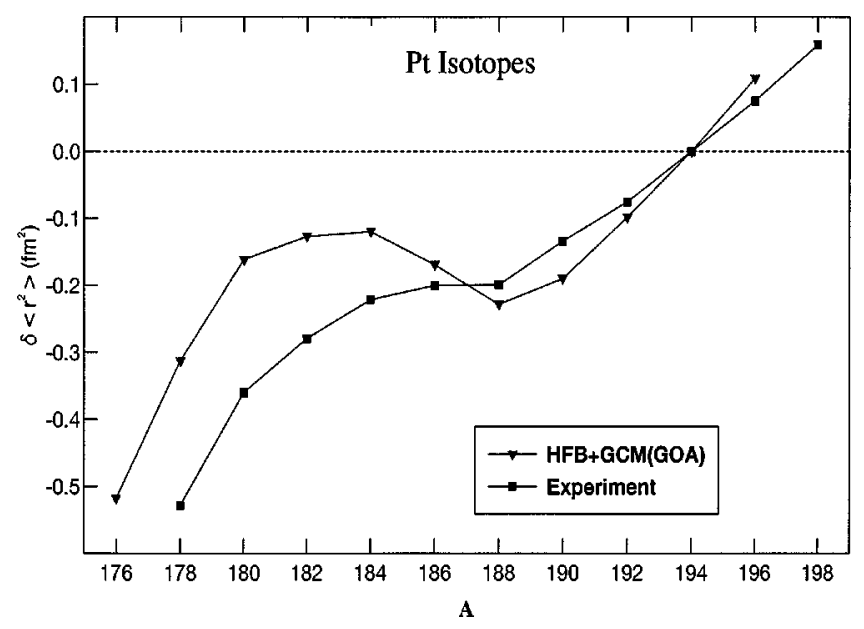

FIG. 5. Measured $\delta\left\langle r_{c}^{2}\right\rangle$ in the even platinum isotopes as shown in Fig. 2 compared with the microscopic Hartree-Fock-Bogoliubov calculations in the configuration mixing approximation. 


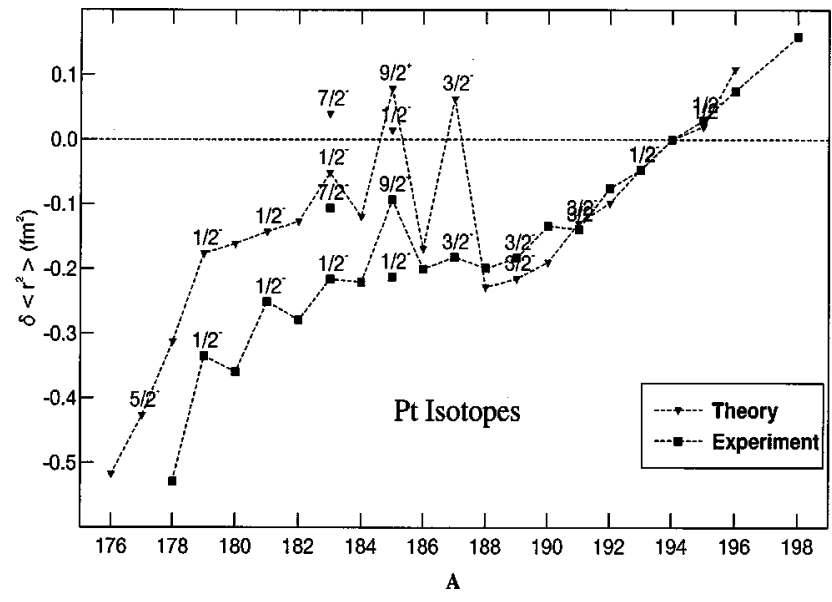

FIG. 6. $\delta\left\langle r_{c}^{2}\right\rangle$ value measured in the platinum isotopes as shown in Fig. 2, compared with our microscopic calculations for ground states and isomers.

have shown that these states correspond to an axially prolate shape. Some theoretical arguments have already been given while discussing the oblate-prolate PEC differences for odd nuclei (see Fig. 4). In this context, using two different theoretical methods to treat separately the even-even and the even-odd nuclei imposes the definition of two sets of $\delta\left\langle r_{c}^{2}\right\rangle_{\text {th }}$ values, namely, one for the even-even isotopes $\left(\delta\left\langle r_{c}^{2}\right\rangle_{\text {th }}^{194, A}\right)$ and the other for the even-odd nuclei $\left(\delta\left\langle r_{c}^{2}\right\rangle_{\text {th }}^{193, A}\right)$. For convenience, the comparison shown in Fig. 6 for both even and odd nuclei is actually performed using $\delta\left\langle r_{c}^{2}\right\rangle_{\text {th }}^{193, A}$ $+\delta\left\langle r_{c}^{2}\right\rangle_{\text {expt }}^{194,193}$ for all the odd nuclei.

As can be seen, our calculations reproduce the main pattern of the measurements, especially the odd-even staggering, except for $A>188$ where the very small differences between measurements and calculations are not significant considering the accuracy of our microscopic models. Furthermore, the $\delta\left\langle r_{c}^{2}\right\rangle$ value predicted for the ${ }^{187} \mathrm{Pt}$ nucleus is too large because ignoring the rotation-vibration coupling in this shape transition nucleus is probably too crude an approximation. Also the staggering seems to disappear in the vicinity of $A=180$. This is related to the $\delta\left\langle r_{c}^{2}\right\rangle$ calculations for the even Pt isotopes which in this case are too large (see Fig. 5). Finally, the isomeric shifts measured and calculated in ${ }^{185} \mathrm{Pt}$ and ${ }^{183} \mathrm{Pt}$ are in good agreement. This result can easily be interpreted considering that the deformations of the $7 / 2^{-}\left({ }^{183} \mathrm{Pt}^{m}\right)$ and $9 / 2^{+}\left({ }^{185} \mathrm{Pt}\right)$ states are larger than that of $1 / 2^{-}$ground or isomeric states (see Figs. 4 and 6). Although $\beta$ is overestimated in the calculations, the experimental $\delta\langle\beta\rangle$ values $(\sim 0.02)$ are well reproduced in both nuclei $\left(\delta\langle\beta\rangle_{\text {th }}\right.$ $=0.01)$.

\section{CONCLUSION}

Hyperfine structure splittings and isotope shifts have been measured for the very-neutron-deficient platinum isotopes from $A=185$ to $A=178$ by resonance ionization spectroscopy on a secondary atomic beam produced by laser desorption. The mean square charge radius variation and nuclear moments were thus determined. The quadrupole moments were extracted for ${ }^{183} \mathrm{Pt}^{m}$ and ${ }^{185} \mathrm{Pt}^{g}$ from which we can obtain the $\beta$ deformation assuming axial symmetry. This parameter is in accordance with $\left\langle\beta^{2}\right\rangle^{1 / 2}$ extracted from $\delta\left\langle r_{c}^{2}\right\rangle$ which means that these nuclei are axially prolate. For the other odd nuclei, the values of the experimental magnetic moments are compatible with those calculated assuming axial symmetry. We have performed Hartree-FockBogolyubov calculations to interpret the charge radius evolution and especially the inverted odd-even staggering between $A=186$ and $A=178$. The PECs obtained assuming axial symmetry show much smaller oblate-prolate energy differences for the even-even Pt isotopes than for the oddeven ones. This indicates again that the triaxial degree of freedom should play an important role for the even isotopes and a minor role for the odd ones. This justifies the comparison of the experimental $\delta\left\langle r_{c}^{2}\right\rangle$ results with axial calculations for the odd isotopes and with triaxial calculations for the even ones. The general trend of the $\delta\left\langle r_{c}^{2}\right\rangle$ evolution along the platinum isotope chain is reproduced, particularly the kink appearing at mass 188 and the deformation drop for the $A$ $\leqslant 178$ masses. Moreover, the $\delta\left\langle r_{c}^{2}\right\rangle$ values measured between the ground and isomeric states in both ${ }^{183} \mathrm{Pt}$ and ${ }^{185} \mathrm{Pt}$ are well reproduced by the axial calculations. Therefore, the even isotopes appear to be triaxial (the predicted $\gamma$ value varying from $33^{\circ}$ for ${ }^{196} \mathrm{Pt}$ to $12^{\circ}$ for ${ }^{180} \mathrm{Pt}$ ) while the light odd ones seem to be axially prolate. This switching from triaxial to axial shape is accompanied by an increase of the $\beta$ deformation parameter in the odd isotopes. Thus, the inverted odd-even staggering observed for the light Pt isotopes can result from alternating shape transitions and can be considered as the signature of a deformation change between the even-even and the odd-even isotopes.

\section{ACKNOWLEDGMENT}

We would like to thank Dr. Jean Libert for fruitful discussions.
[1] J. Bonn, G. Huber, H.-J. Kluge, and E. W. Otten, Z. Phys. A 276, 203 (1976).

[2] G. Ulm et al., Z. Phys. A 325, 247 (1986).

[3] R. Béraud, M. Meyer, M. G. Desthuilliers, C. Bourgeois, P. Kilcher, J. Letessier, and ISOCELE Collaboration, Nucl. Phys. A284, 221 (1977).

[4] J. D. Cole et al., Phys. Rev. C 16, 2010 (1977).
[5] P. Dabkiewicz, F. Buchinger, H. Fischer, H.-J. Kluge, H. Kremmling, T. Kühl, A. C. Müller, and H. A. Schuessler, Phys. Lett. 82B, 199 (1979).

[6] A. Visvanathan, E. F. Zganjar, J. L. Wood, R. W. Fink, L. L. Riedinger, and F. E. Turner, Phys. Rev. C 19, 282 (1979).

[7] B. Roussière, C. Bourgeois, P. Kilcher, J. Sauvage, M. G. Porquet, and ISOCELE Collaboration, Nucl. Phys. A504, 511 
(1989).

[8] J. Nyberg et al., Nucl. Phys. A511, 92 (1990).

[9] E. Hagberg, P. G. Hansen, P. Hornshøj, B. Jonson, S. Mattson, P. Tidemand-Petersson, and ISOLDE Collaboration, Phys. Lett. 78B, 44 (1978).

[10] J. Sauvage, D. Hojman, F. Ibrahim, B. Roussière, P. Kilcher, F. Le Blanc, J. Oms, J. Libert, and ISOCELE Collaboration, Nucl. Phys. A592, 221 (1995).

[11] G. D. Dracoulis, A. E. Stuchbery, A. P. Byrne, A. R. Poletti, S. J. Poletti, J. Gerl, and R. A. Bark, J. Phys. G 12, L97 (1986).

[12] G. D. Dracoulis, B. Fabricius, A. E. Stuchbery, A. O. Macchiavelli, W. Korten, F. Azaeiz, E. Rubel, M. A. Deleplanque, R. M. Diamond, and F. S. Stephens, Phys. Rev. C 44, R1246 (1991).

[13] D. Seweryniak et al., Phys. Rev. C 58, 2710 (1998).

[14] J. K. P. Lee et al., Phys. Rev. C 38, 2985 (1988).

[15] H. T. Duong et al., Phys. Lett. B 217, 401 (1989).

[16] T. Hilberath, St. Becker, G. Bollen, H.-J. Kluge, G. Passler, J. Rikovska, R. Wyss, and ISOLDE Collaboration, Z. Phys. A 342, 1 (1992).

[17] P. Kilcher et al., Nucl. Instrum. Methods Phys. Res. B 70, 537 (1992).

[18] J. Wood, K. Heyde, W. Nazarewicz, M. Huyse, and P. van Duppen, Phys. Rep. 215, 103 (1992).

[19] K. Heyde, P. van Isacker, M. Waroquier, J. L. Wood, and R. A. Meyer, Phys. Rep. 102, 291 (1983).

[20] M. K. Harder, K. T. Tang, and P. van Isacker, Phys. Lett. B 405, 25 (1997).

[21] R. Bengtsson, T. Bengtsson, J. Dudek, G. Leander, W. Nazarewicz, and J. Zhang, Phys. Lett. B 183, 1 (1987).

[22] B. Cederwall et al., Z. Phys. A 337, 283 (1990).

[23] F. Le Blanc et al., in Proceedings of the 8th International Symposium on Capture Gamma-Ray Spectroscopy and Related
Topics, Fribourg, Switzerland, 1993, edited by J. Kern (World Scientific, Singapore, 1994), p. 1001.

[24] F. Le Blanc et al., Phys. Rev. Lett. 79, 2213 (1997).

[25] S. Büttgenbach, N. Glaeser, B. Roski, and F. Träber, Z. Phys. A 317, 237 (1984).

[26] P. Raghavan, At. Data Nucl. Data Tables 42, 189 (1989).

[27] W. Childs and K. Cheng, Phys. Rev. A 30, 667 (1984).

[28] S. Raman, C. W. Nestor, S. Kahane, and K. H. Bhatt, At. Data Nucl. Data Tables 42, 1 (1989).

[29] K. Heilig and A. Steudel, At. Data Nucl. Data Tables 14, 613 (1974).

[30] W. Neu, G. Passler, G. Sawatzky, R. Winkler, and H.-J. Kluge, Z. Phys. D 7, 193 (1987).

[31] E. C. Seltzer, Phys. Rev. 188, 1916 (1969).

[32] S. A. Ahmad, W. Klempt, C. Ekström, R. Neugart, K. Wendt, and ISOLDE Collaboration, Z. Phys. A 321, 35 (1985).

[33] J. Libert, M. Meyer, and P. Quentin, Phys. Rev. C 25, 586 (1982).

[34] M. G. Porquet, J. Sauvage, M. Meyer, and P. Quentin, Nucl. Phys. A451, 365 (1986).

[35] D. Zawischa, Phys. Lett. 155B, 309 (1985).

[36] J. Dechargé and D. Gogny, Phys. Rev. C 21, 1568 (1980).

[37] M. Girod, P. Dessagne, M. Bernas, M. Langevin, F. Pougheon, and P. Roussel, Phys. Rev. C 37, 2600 (1988).

[38] M. Girod and B. Grammaticos, Phys. Rev. C 27, 2317 (1983).

[39] P. Ring and P. Schuck, in The Nuclear Many-Body Problem (Springer, Berlin, 1980), p. 398.

[40] D. L. Hill and J. A. Wheeler, Phys. Rev. 89, 1102 (1953); J. J. Griffin and J. A. Wheeler, ibid. 108, 311 (1957).

[41] B. Giraud and B. Grammaticos, Nucl. Phys. A233, 373 (1974); A255, 141 (1975); M. Girod and B. Grammaticos, ibid. A330, 40 (1979), and references therein.

[42] L. Esser, U. Neuneyer, R. F. Casten, and P. von Brentano, Phys. Rev. C 55, 206 (1997). 